International Journal of Pure and Applied Mathematics

Volume 116 No. 1 2017, 91-96

ISSN: 1311-8080 (printed version); ISSN: 1314-3395 (on-line version)

url: http://www.ijpam.eu

doi: 10.12732 /ijpam.v116i1.8

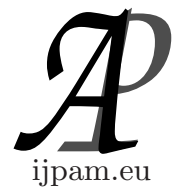

\title{
LINEAR CANONICAL TRANSFORM FOR INTEGRABLE BOEHMIANS
}

\author{
Pravinkumar V. Dole ${ }^{1 \S}$, S.K. Panchal ${ }^{2}$ \\ Department of Mathematics \\ Dr. Babasaheb Ambedkar Marathwada University \\ Aurangabad, 431004 (M.S.), INDIA
}

\begin{abstract}
In this paper we extend the linear canonical transform to class of integrable Boehmians by using new definition of convolution defined in [3]. Further, we prove that the extended linear canonical transform have expected properties like linear, one-to-one, onto and continuous from one Boehmian space to another Boehmian space and obtained some basic properties.
\end{abstract}

AMS Subject Classification: 44A35, 44A40, 46F12, 46F99

Key Words: linear canonical transform, generalized functions, convolutions, boehmians

\section{Introduction}

Boehmian is a new class of generalized functions introduced by Boehme [1] which open the new door to area of research in mathematics $[4,5,7,8]$. The construction of Boehmians is given by Mikusinski and Mikusinski. Mikusinski has studied Fourier transform of integrable Boehmians in [6]. Zayed and Abhishek singh extend the fractional Fourier transform to class of integrable Boehmians in [11,9] respectively. Bhosale and Chaudhary [2] extended the fractional Fourier transform to distributions of compact support. The Fourier transform and fractional Fourier transform are the special cases of linear canonical transform $(\mathrm{LCT})[3]$. Let $\mathcal{L}^{1}(\mathbb{R})$ be the space of all complex valued absolutely integrable functions on $\mathbb{R}$ with norm $\|f\|_{1}=\int_{\mathbb{R}}|f(t)| d t \leq M$, for some $M>0$.

\footnotetext{
Received: February 24, 2017

Revised: June 21, 2017

Published: $\quad$ August 29, 2017

$\S_{\text {Correspondence author }}$
}

(c) 2017 Academic Publications, Ltd. url: www.acadpubl.eu 
The linear canonical transform of $f \in \mathcal{L}^{1}(\mathbb{R})$, is define as

$$
F_{A}=\mathcal{L}_{A}[f(t)](u)= \begin{cases}\sqrt{\frac{1}{2 \pi i b}} \int_{-\infty}^{\infty} e^{\frac{i}{2}\left[\frac{a}{b} t^{2}-\frac{2}{b} u t+\left(\frac{d}{b}\right) u^{2}\right]} f(t) d t, & \text { for } b \neq 0 \\ \sqrt{d} e^{\frac{i}{2} c d u^{2}} f(d u), & \text { for } b=0\end{cases}
$$

where $\mathcal{L}_{A}$ is the unitary linear canonical operator and $A=(a, b, c, d), a, b, c, d$ are real number satisfying $a d-b c=1$. The inverse transform for linear canonical transform is given by a linear canonical transform having parameter $A^{-1}=$ $(d,-b,-c, a)$

$$
f(t)= \begin{cases}\sqrt{\frac{1}{-2 \pi i b}} \int_{-\infty}^{\infty} e^{\frac{i}{2}\left[\left(\frac{-d}{b}\right) u^{2}+\left(\frac{2}{b}\right) u t-\left(\frac{a}{b}\right) t^{2}\right]} F_{A}(u) d u, & \text { for } b \neq 0, \\ \sqrt{a} e^{\frac{i}{2} c a u^{2}} f(a u), & \text { for } b=0 .\end{cases}
$$

Definition 1.1. [3] Let $W(t, \tau)=e^{i \tau(\tau-t) \frac{a}{b}}$ be the weight function. For any two functions $f$ and $g$ the convolution operation $*^{A}$ is defined as,

$$
h(t)=\left(f *^{A} g\right)(t)=\int_{-\infty}^{\infty} f(\tau) g(t-\tau) W(t, \tau) d \tau
$$

Theorem 1.1. [3] (New Convolution Theorem) Let $h(t)=\left(f *^{A} g\right)(t)$ and $H_{A}(u), F_{A}(u), G_{A}(u)$ denote the linear canonical transform of $h(t), f(t)$ and $g(t)$ respectively, then

$$
H_{A}(u)=\sqrt{2 i \pi b} e^{-i\left(\frac{d u^{2}}{2 b}\right)} F_{A}(u) G_{A}(u) .
$$

\section{Preliminary Results}

In this section we obtain some results which are required to construct space of integrable Boehmians.

Lemma 2.1. Let $f, g \in \mathcal{L}^{1}(\mathbb{R})$, then $\left(f *^{A} g\right)$ is also in $\mathcal{L}^{1}(\mathbb{R})$.

Lemma 2.2. The space $\left(\mathcal{L}^{1}(\mathbb{R}), *^{A}\right)$ is a commutative semi group.

Definition 2.1. Let $\triangle$ denote the collection of all sequences of continuous real functions $\left\{\delta_{n}\right\}$ from $\mathcal{L}^{1}(\mathbb{R})$ having compact support, with the following conditions:

(i) $\int_{\mathbb{R}} e^{i \frac{a t^{2}}{2 b}} \delta_{n}(t) d t=1, \forall n \in \mathbb{N}$, 
(ii) $\quad\left\|\delta_{n}\right\|_{1} \leq M, \forall n \in \mathbb{N}$ for some $M>0$,

(iii) $\lim _{n \rightarrow \infty} \int_{|t|>\epsilon}\left|\delta_{n}(t)\right| d t \longrightarrow 0$, for each $\epsilon>0$. The members of $\triangle$ are called delta sequences. For example, let $a, b \in \mathbb{R} ; b \neq 0$, consider the sequence

$$
\delta_{n}(t)= \begin{cases}e^{-i \frac{a t^{2}}{2 b}} t, & \text { for } 0 \leq t \leq \frac{1}{n} \\ e^{-i \frac{a t^{2}}{2 b}} n^{2}\left(\frac{2}{n}-t\right), & \text { for } \frac{1}{n} \leq t \leq \frac{2}{n} \\ 0, & \text { otherwise }\end{cases}
$$

Lemma 2.3. Let $\left\{\varphi_{n}\right\},\left\{\psi_{n}\right\} \in \triangle$ then $\left\{\varphi_{n} *^{A} \psi_{n}\right\} \in \triangle$.

Lemma 2.4. Let $f \in \mathcal{L}^{1}(\mathbb{R})$ and $\left\{\psi_{n}\right\} \in \triangle$ then $f *^{A} \psi_{n} \longrightarrow f$ as $n \longrightarrow \infty$ in $\mathcal{L}^{1}(\mathbb{R})$.

\section{LCT For Integrable Boehmians}

A pair of sequences $\left(f_{n}, \varphi_{n}\right)$ is called a quotient of the sequences, denoted by $f_{n} / \varphi_{n}$, where $f_{n} \in \mathcal{L}^{1}(\mathbb{R})(n \in \mathbb{N}),\left\{\varphi_{n}\right\}$ is a delta sequence and $f_{m} *^{A} \varphi_{n}=$ $f_{n} *^{A} \varphi_{m}$ holds $\forall m, n \in \mathbb{N}$. Two quotient of sequences $f_{n} / \varphi_{n}$ and $g_{n} / \psi_{n}$ are equivalent if $f_{n} *^{A} \psi_{n}=g_{n} *^{A} \varphi_{n} \forall n \in \mathbb{N}$. The equivalence class of quotient of sequences is called an integrable Boehmian. The space of all integrable Boehmians is denoted by $\mathcal{B}_{\mathcal{L}^{1}}=\mathcal{B}_{\mathcal{L}^{1}}\left(\mathcal{L}^{1}(\mathbb{R}), \triangle, *^{A}\right)$ and the members of $\mathcal{B}_{\mathcal{L}^{1}}$ are denoted by $F=\left[f_{n} / \varphi_{n}\right]$. The function $f \in \mathcal{L}^{1}(\mathbb{R})$ can be identified with the Boehmian $\left[\left(f *^{A} \delta_{n}\right) / \delta_{n}\right]$, where $\left\{\delta_{n}\right\}$ is the delta sequence. If $F=\left[f_{n} / \varphi_{n}\right]$, then $f *^{A} \delta_{n}=f_{n} \in \mathcal{L}^{1}(\mathbb{R}) \forall n \in \mathbb{N}$.

Definition 3.1. A sequence of Boehmians $F_{n}$ is called $\Delta$-convergent to a Boehmian $F\left(\Delta-\lim F_{n}=F\right)$ if there exist a delta sequence $\left\{\delta_{n}\right\}$ such that $\left(F_{n}-F\right) *^{A} \delta_{n} \in \mathcal{L}^{1}(\mathbb{R})$, for every $n \in \mathbb{N}$ and that $\left\|\left(F_{n}-F\right) *^{A} \delta_{n}\right\|_{1} \rightarrow 0$ as $n \rightarrow \infty$.

Definition 3.2. A sequence of Boehmians $F_{n}$ is called $\delta$-convergent to a Boehmian $F\left(\delta-\lim F_{n}=F\right)$ if there exist a delta sequence $\left\{\delta_{n}\right\}$ such that $F_{n} *^{A} \delta_{k} \in \mathcal{L}^{1}(\mathbb{R})$ and $F *^{A} \delta_{k} \in \mathcal{L}^{1}(\mathbb{R})$ for every $n, k \in \mathbb{N}$ and that $\left\|\left(F_{n}-F\right) *^{A} \delta_{k}\right\|_{1} \rightarrow 0$ as $n \rightarrow \infty$ for each $k \in \mathbb{N}$.

Let $\left\{\delta_{n}\right\}$ is a delta sequence, then $\delta_{n} / \delta_{n}$ represents an integrable Boehmian. Since the Boehmian $\left[\delta_{n} / \delta_{n}\right]$ corresponds to Dirac delta distribution $\delta$, all the derivative of $\delta$ are also integrable Boehmian. If $\left\{\delta_{n}\right\}$ is infinitely differentiable and bounded, then the $k^{\text {th }}$ derivative of $\delta$ is define by $\delta^{(k)}=\left[\delta_{n}^{(k)} / \delta_{n}\right] \in \mathcal{B}_{\mathcal{L}^{1}}$, for each $k \in \mathbb{N}$. The $k^{\text {th }}$ derivative of Boehmian $F \in \mathcal{B}_{\mathcal{L}^{1}}$ is define by $F^{(k)}=$ $F *^{A} \delta^{(k)}$. 
Lemma 3.1. Let $\Delta-\lim F_{n}=F$ in $\mathcal{B}_{\mathcal{L}^{1}}$ then $\Delta-\lim F_{n}^{(k)}=F^{(k)}$ for $\forall k \in \mathbb{N}$ in $\mathcal{B}_{\mathcal{L}^{1}}$.

Lemma 3.2. Let $\Delta-\lim _{n \rightarrow \infty} F_{n}=F$ and $\Delta-\lim _{n \rightarrow \infty} G_{n}=G$ in $\mathcal{B}_{\mathcal{L}^{1}}$ then $\Delta-\lim _{n \rightarrow \infty} F_{n} *^{A} G_{n}=F *^{A} G$ in $\mathcal{B}_{\mathcal{L}^{1}}$.

The scalar multiplication, addition and convolution in $\mathcal{B}_{\mathcal{L}^{1}}$ are define as,

$$
\begin{aligned}
\lambda\left[f_{n} / \varphi_{n}\right] & =\left[\lambda f_{n} / \varphi_{n}\right] \\
{\left[f_{n} / \varphi_{n}\right]+\left[g_{n} / \psi_{n}\right] } & =\left[\left(f_{n} *^{A} \psi_{n}+g_{n} *^{A} \varphi_{n}\right) / \varphi_{n} *^{A} \psi_{n}\right] \\
{\left[f_{n} / \varphi_{n}\right] *^{A}\left[g_{n} / \psi_{n}\right] } & =\left[\left(f_{n} *^{A} g_{n}\right) /\left(\varphi_{n} *^{A} \psi_{n}\right)\right]
\end{aligned}
$$

Lemma 3.3. Let $\left\{\delta_{n}\right\} \in \triangle$ then $\mathcal{L}_{A}\left(\delta_{n}\right)$ converges uniformly on each compact set to a constant function 1 .

Let $\triangle_{0}=\left\{\mathcal{L}_{A}\left(\delta_{n}\right) ;\left\{\delta_{n}\right\} \in \triangle\right\}$ be the space of complex valued functions on $\mathbb{R}$, the operation $\cdot$ is point wise multiplication and $C_{0}(\mathbb{R})$ be the space of all continuous functions vanishing at infinity on $\mathbb{R}$ then we construct the another space of Boehmians, denoted by $\mathcal{B}=\mathcal{B}\left(\mathcal{L}^{1}(\mathbb{R}), C_{0}(\mathbb{R}) \cap \mathcal{L}^{1}(\mathbb{R}), \cdot, \triangle_{0}\right)$. This is the range of linear canonical transform on $\mathcal{B}_{\mathcal{L}^{1}}$ and each element of $\mathcal{B}$ is denoted by $\mathcal{L}_{A}\left(f_{n}\right) / \mathcal{L}_{A}\left(\delta_{n}\right)$ for all $n \in \mathbb{N}$, where $\left\{f_{n}\right\} \in \mathcal{L}^{1}(\mathbb{R})$ for $n \in \mathbb{N}$.

Lemma 3.4. Let $f, g \in \mathcal{L}^{1}(\mathbb{R}) ; \varphi, \psi \in C_{0}(\mathbb{R})$ and $\lambda \in \mathbb{C}$ then (i) $f \cdot \varphi \in$ $\mathcal{L}^{1}(\mathbb{R})($ ii) $(f+g) \cdot \varphi=f \cdot \varphi+f \cdot \varphi($ iii) $(\lambda f) \cdot \varphi=\alpha(f \cdot \varphi)$ (iv) $f \cdot(\varphi \cdot \psi)=(f \cdot \varphi) \cdot \psi$.

Lemma 3.5. Let $f_{n} \rightarrow f$ as $n \rightarrow \infty$ in $\mathcal{L}^{1}(\mathbb{R})$ and $\varphi \in C_{0}(\mathbb{R})$ then $f_{n} \cdot \varphi \rightarrow f \cdot \varphi$ in $\mathcal{L}^{1}(\mathbb{R})$.

Lemma 3.6. Let $f_{n} \rightarrow f$ as $n \rightarrow \infty$ in $\mathcal{L}^{1}(\mathbb{R})$ and $\mathcal{L}_{A}\left(\varphi_{n}\right) \in \triangle_{0}$ then $f_{n} \cdot \mathcal{L}_{A}\left(\varphi_{n}\right) \rightarrow f$ in $\mathcal{L}^{1}(\mathbb{R})$.

Lemma 3.7. If $\mathcal{L}_{A}\left(\varphi_{n}\right), \mathcal{L}_{A}\left(\psi_{n}\right) \in \triangle_{0}$ then $\mathcal{L}_{A}\left(\varphi_{n}\right) \cdot \mathcal{L}_{A}\left(\psi_{n}\right) \in \triangle_{0}$.

Definition 3.3. Let $\left\{f_{n}\right\} \in \mathcal{L}^{1}(\mathbb{R})$ and $\left\{\delta_{n}\right\} \in \triangle$ for $n \in \mathbb{N}$, we define the linear canonical transform $\mathcal{L}_{A}: \mathcal{B}_{\mathcal{L}^{1}} \longrightarrow \mathcal{B}$ as

$$
\mathcal{L}_{A}\left[f_{n} / \delta_{n}\right]=\mathcal{L}_{A}\left(f_{n}\right) / \mathcal{L}_{A}\left(\delta_{n}\right) \quad \text { for } \quad\left[f_{n} / \delta_{n}\right] \in \mathcal{B}_{\mathcal{L}^{1}}
$$

The linear canonical transform on $\mathcal{B}_{\mathcal{L}^{1}}$ is well defined. Indeed if $\left[f_{n} / \delta_{n}\right] \in$ $\mathcal{B}_{\mathcal{L}^{1}}$, then $f_{n} *^{A} \delta_{m}=f_{m} *{ }^{A} \delta_{n}$ for all $m, n \in \mathbb{N}$. Applying the linear canonical transform on both sides, we get $\mathcal{L}_{A}\left(f_{n}\right) \mathcal{L}_{A}\left(\delta_{m}\right)=\mathcal{L}_{A}\left(f_{m}\right) \mathcal{L}_{A}\left(\delta_{n}\right)$ for all $m, n \in$ $\mathbb{N}$ and hence $\mathcal{L}_{A}\left(f_{n}\right) / \mathcal{L}_{A}\left(\delta_{n}\right) \in \mathcal{B}$. Further if $\left[f_{n} / \psi_{n}\right]=\left[g_{n} / \delta_{n}\right] \in \mathcal{B}_{\mathcal{L}^{1}}$ then we have $f_{n} *^{A} \delta_{n}=g_{n} *^{A} \psi_{n}$ for all $n \in \mathbb{N}$. Again applying the linear canonical transform on both sides, we get $\mathcal{L}_{A}\left(f_{n}\right) \mathcal{L}_{A}\left(\delta_{n}\right)=\mathcal{L}_{A}\left(g_{n}\right) \mathcal{L}_{A}\left(\psi_{n}\right)$ for all $n \in \mathbb{N}$. i.e. $\mathcal{L}_{A}\left(f_{n}\right) / \mathcal{L}_{A}\left(\psi_{n}\right)=\mathcal{L}_{A}\left(g_{n}\right) / \mathcal{L}_{A}\left(\delta_{n}\right)$ in $\mathcal{B}$. 
Lemma 3.8. Let $\left[f_{n} / \varphi_{n}\right] \in B_{\mathcal{L}^{1}}$ then the linear canonical transform of the sequence

$$
\mathcal{L}_{A}\left[f_{n}\right](u)=\sqrt{\frac{1}{2 \pi i b}} e^{\frac{i}{2}\left(\frac{d}{b}\right) u^{2}} \int_{-\infty}^{\infty} e^{\frac{-i}{b} u t} e^{\frac{i}{2} \frac{a}{b} t^{2}} f_{n}(t) d t
$$

converges uniformly on each compact set in $\mathbb{R}$.

Proof. Let $\left(\delta_{n}\right)$ is delta sequence, then $\mathcal{L}_{A}\left(\delta_{n}\right)$ converges uniformly on each compact set to a constant function 1 . Hence, for each compact set $M$ of $\mathbb{R}$, $\mathcal{L}_{A}\left[\delta_{m}\right]>0$ on $M$, for almost all $m \in \mathbb{N}$. Moreover, $\mathcal{L}_{A}\left(f_{n}\right)=\mathcal{L}_{A}\left(f_{n}\right) \frac{\mathcal{L}_{A}\left(\delta_{m}\right)}{\mathcal{L}_{A}\left(\delta_{m}\right)}=$ $\frac{\mathcal{L}_{A}\left(f_{m}\right)}{\mathcal{L}_{A}\left(\delta_{m}\right)} \mathcal{L}_{A}\left(\delta_{n}\right)$, as $n \rightarrow \infty$ we get $\mathcal{L}_{A}\left(f_{n}\right) \rightarrow \frac{\mathcal{L}_{A}\left(f_{m}\right)}{\mathcal{L}_{A}\left(\delta_{m}\right)}$, on each compact set $M$.

Definition 3.4. In view of the above lemma, the linear canonical transform of an integrable Boehmian in a space of continuous functions on $\mathbb{R}$ can be defined as,

$$
\mathcal{L}_{A}[F]=\lim _{n \rightarrow \infty} \mathcal{L}_{A}\left(f_{n}\right)
$$

Now, we show that the above definition is well define. Let two Boehmians $\left[f_{n} / \varphi_{n}\right]$ and $\left[g_{n} / \psi_{n}\right]$ are the same but representation is different then $f_{n} *^{A} \psi_{n}=$ $g_{n} *^{A} \varphi_{n}$ for all $n \in \mathbb{N}$ taking linear canonical transform on both side we have

$$
\mathcal{L}_{A}\left(f_{n}\right)(\mu) \mathcal{L}_{A}\left(\psi_{n}\right)(\mu)=\mathcal{L}_{A}\left(g_{n}\right)(\mu) \mathcal{L}_{A}\left(\varphi_{n}\right)(\mu)
$$

which implies that $\lim _{n \rightarrow \infty} \mathcal{L}_{A}\left(f_{n}\right)=\lim _{n \rightarrow \infty} \mathcal{L}_{A}\left(g_{n}\right)$. Hence $\mathcal{L}_{A}\left[f_{n} / \varphi_{n}\right]=$ $\mathcal{L}_{A}\left[g_{n} / \psi_{n}\right]$.

Theorem 3.1. The linear canonical transform $\mathcal{L}_{A}: \mathcal{B}_{\mathcal{L}^{1}} \longrightarrow \mathcal{B}$ is consistent with $\mathcal{L}_{A}: \mathcal{L}^{1}(\mathbb{R}) \longrightarrow \mathcal{L}^{1}(\mathbb{R})$.

Theorem 3.2. The linear canonical transform $\mathcal{L}_{A}: \mathcal{B}_{\mathcal{L}^{1}} \longrightarrow \mathcal{B}$ is a bijection.

we skip the proofs of above two theorems.

Theorem 3.3. Let $F, G \in \mathcal{B}_{\mathcal{L}^{1}}$ then:

(a) $\mathcal{L}_{A}(\lambda F+G)=\lambda \mathcal{L}_{A}(F)+\mathcal{L}_{A}(G)$, for any complex $\lambda$,

(b) $\mathcal{L}_{A}\left(F *^{A} G\right)=\mathcal{L}_{A}(F) \mathcal{L}_{A}(G)$,

(c) $\mathcal{L}_{A}\left(e^{i k t} F\right)(u)=e^{\frac{i d k(2 u-b k)}{2}} \mathcal{L}_{A}(F)(u-b k)$, for $k \in \mathbb{R}$,

(d) $\mathcal{L}_{A}(F(t+\tau)(u))=e^{\frac{i u \tau}{b}} e^{\frac{i a \tau^{2}}{2 b}} \mathcal{L}_{A}\left(e^{\frac{-i a x \tau}{b}} F(x)\right)(u)$,

(e) $\mathcal{L}_{A}\left(F^{(k)}\right)(u)=\left(\frac{i u}{b}\right)^{k} \mathcal{L}_{A}(F)(u)$ for all $k \in \mathbb{N}$, 
(f) If $\mathcal{L}_{A}(F)=0$, then $F=0$,

(g) Let $F_{n}, F \in \mathcal{B}_{\mathcal{L}^{1}}$, if $\Delta-\lim F_{n}=F$ then $\mathcal{L}_{A}\left(F_{n}\right) \rightarrow \mathcal{L}_{A}(F)$ uniformly on each compact set of $\mathbb{R}$.

Proof. The proofs of the above properties (a)-(f) are straightforward. Now we prove $(\mathrm{g})$, let $\left\{\delta_{m}\right\}$ be a delta sequence such that $F_{n} *^{A} \delta_{m}, F *^{A} \delta_{m} \in \mathcal{L}^{1}(\mathbb{R})$ for all $n, m \in \mathbb{N}$ and $\left\|\left(F_{n}-F\right) *^{A} \delta_{m}\right\|_{1} \rightarrow 0$ as $n \rightarrow \infty$ for each $m \in \mathbb{N}$. Let $M$ be a compact set in $\mathbb{R}$ then $\mathcal{L}_{A}\left(\delta_{m}\right)>0$ on $M$ for all most $m \in \mathbb{N}$. Since $\mathcal{L}_{A}\left(\delta_{m}\right)$ is a continuous function and $\mathcal{L}_{A}\left(F_{n}\right) *{ }^{A} \mathcal{L}_{A}\left(\delta_{m}\right)-\mathcal{L}_{A}(F) *{ }^{A} \mathcal{L}_{A}\left(\delta_{m}\right)=$ $\left(\left(\mathcal{L}_{A}\left(F_{n}\right)-\mathcal{L}_{A}(F)\right) *{ }^{A} \mathcal{L}_{A}\left(\delta_{m}\right)\right)$, implies $\left\|\left(\mathcal{L}_{A}\left(F_{n}\right)-\mathcal{L}_{A}(F)\right) *{ }^{A} \mathcal{L}_{A}\left(\delta_{m}\right)\right\|_{1} \rightarrow 0$ as $n \rightarrow \infty$, thus $\mathcal{L}_{A}\left(F_{n}\right) \rightarrow \mathcal{L}_{A}(F)$ uniformly on $M$.

\section{References}

[1] T.K. Boehme, The support of Mikusinski operators, Trans. Amer. Math. Soc., 176 (1973), 319-334.

[2] B.N. Bhosale, M.S. Chaudhary, Fractional Fourier transform of distributions of compact support, Bull. Cal. Math. Soc., 94, No. 5 (2002), 349-358.

[3] Deyun Wei, Qiwen Ran and Yong Li, New convolution theorem for the linear canonical transform and its translation invariance property, Optik, 123 (2012), 1478-1481, doi: 10.1016/j.ijleo.2011.08.054.

[4] J. Mikusinski, P. Mikusinski, Quotients de suites et leurs applications dans l'analyse fonctionnelle, C.R. Acad. Sci. Paris Ser. I Math., 293 (1981), 463-464.

[5] P. Mikusinski, Convergence of Boehmians, Japan. J. Math, 9, No. 1 (1983), 159-179.

[6] P. Mikusinski, Fourier transform for integrable Boehmians, Rocky Moun-tain J. Math., 17, No. 3 (1987), 577-582.

[7] P. Mikusinski, Boehmians and generalized functions, Acta. Math. Hungarica, 51 (1988), 159-179.

[8] P. Mikusinski, Transforms of Boehmians, Different Aspects of Differentiability Dissertationes Mathematicae, 340 (1995), 201-206.

[9] Abhishek Singh, Fractional Fourier transform of a class of Boehmians, International Journal of Pure and Applied Mathematics, 101, No. 3 (2015), 413-420, doi: http://dx.doi.org/10.12732/ijpam.v10113.9.

[10] Walter Rudin, Real and Complex Analysis, Third Edition, McGraw-Hill, New York, 1987.

[11] A.I. Zayed, Fractional Fourier transform of generalized functions, Integ. Trans. Spl. Funct., 7 (1998), 299-312. 